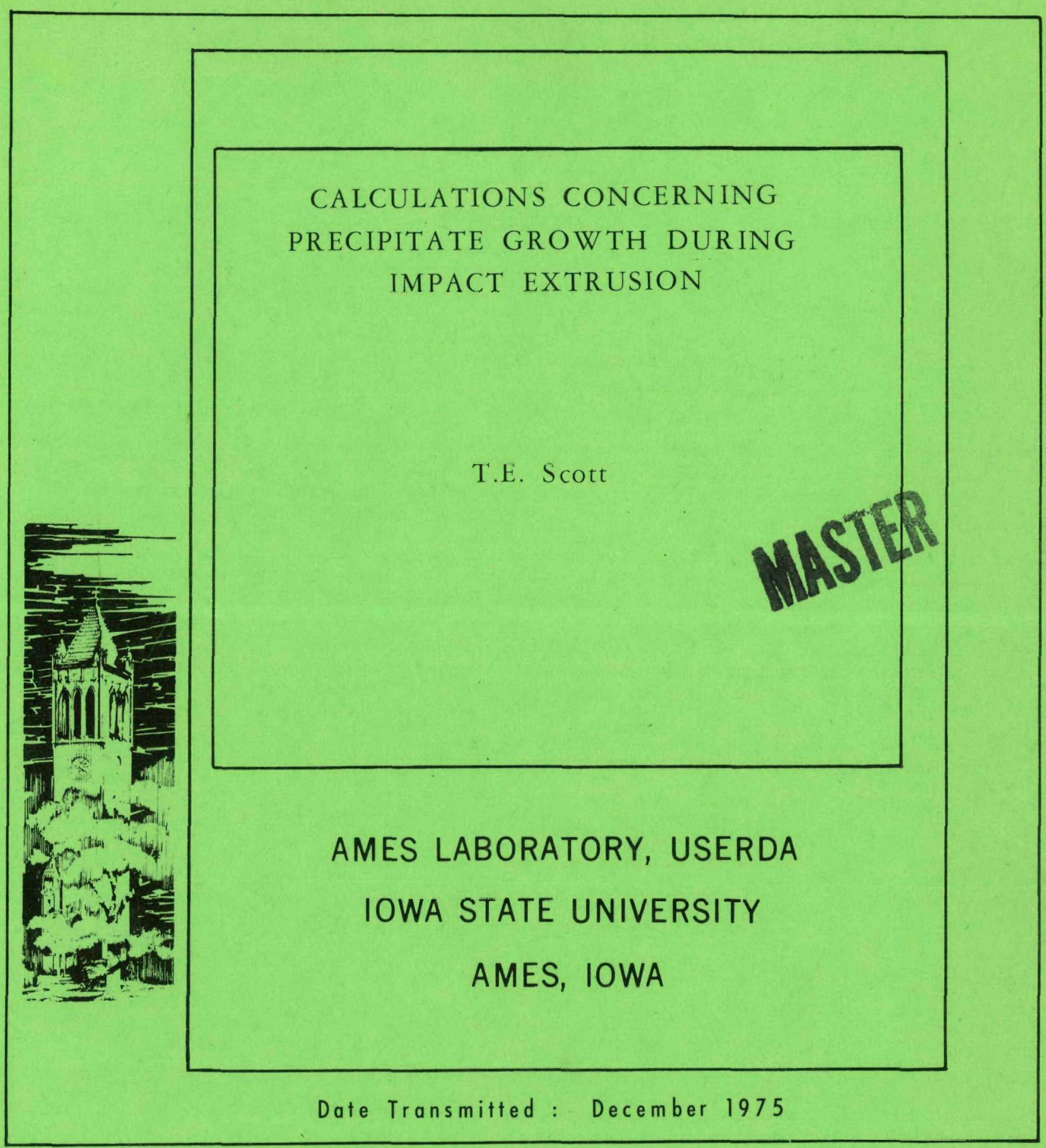

PREPARED FOR THE U. S. ENERGY RESEARCH AND DEVELOPMENT ADMINISTRATION UNDER CONTRACT W-7405-eng-82 


\section{DISCLAIMER}

This report was prepared as an account of work sponsored by an agency of the United States Government. Neither the United States Government nor any agency Thereof, nor any of their employees, makes any warranty, express or implied, or assumes any legal liability or responsibility for the accuracy, completeness, or usefulness of any information, apparatus, product, or process disclosed, or represents that its use would not infringe privately owned rights. Reference herein to any specific commercial product, process, or service by trade name, trademark, manufacturer, or otherwise does not necessarily constitute or imply its endorsement, recommendation, or favoring by the United States Government or any agency thereof. The views and opinions of authors expressed herein do not necessarily state or reflect those of the United States Government or any agency thereof. 


\section{DISCLAIMER}

Portions of this document may be illegible in electronic image products. Images are produced from the best available original document. 


\title{
CALCULATIONS CONCERNING PRECIPITATE GROWTH DURING IMPACT EXTRUSION
}

T. E. Scott

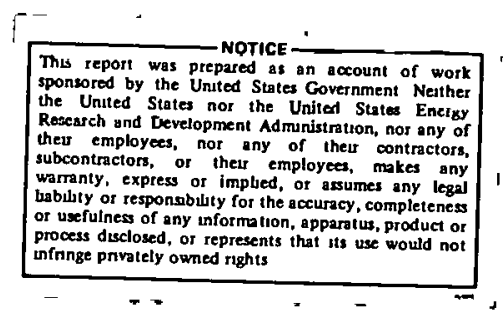

\author{
Ames Laboratory, ERDA \\ Iowa State University \\ Ames, Iowa 50010
}

Date Transmitted: December 1975

PREPARED FOR THE U.S. ENERGY RESEARCH AND

DEVELOPMENT ADMINISTRATION UNDER CONTRACT NO. W-7405-eng-82 
THIS PAGE

WAS INTENTIONALLY

LEFT BLANK 
Distribution List

T. E. Scott 15

Ames Laboratory Library 10 ERDA-TIC 
ABSTRACT

An analysis of carbide particle growth during impact extrusion of $440 \mathrm{~A}$ stainless steel was made and it was concluded from this analysis that diffusion enhancements of the order of 8000 or more would be needed to produce particles with $25 \hat{n}$ radii under such conditions. Deformation-induced excess vacancy enhanced diffusion was examined for the conditions of impact extrusion and it was learned that significant diffusion enhancement would not occur by this mechanism. Finally, dislocation "pipe" diffusion as a possible diffusion enhancement mechanism was evaluated. Although it would appear that "pipe" diffusion is capable of providing large enhancements, the experimental results were contradictory to the behavior predicted by the "pipe" diffusion model.

The overall conclusion from the analyses included herein was that carbide particles cannot grow to physicalily meaningful sizes during impact extrusion. 
INTRODUCTION:

This report effectively is an appendix to a paper entitled "Properties of Steels Ausformed by Impact Extrusion" which will appear in a book to be published by AIME. The book Hot Deformation of Austenite included papers presented in a special session of the same title during the 1975 Fall Meeting of the Metallurgical Society of AIME at Cincinatti, Ohio. One important experimental observation reported in the aforementioned paper was a maximum or peak in the yield strength versus ausforming temperature. Attempts to explain the peak in terms of currently accepted ausform strengthening mechanisms, specifically precipitation during deformation, were negated by arguments concerning the very fast kinetics that would be required during the short deformation intervals characteristic of impact extrusion. The details of the kinetic arguments were too lengthy to include in the manuscript so it was decided to make them available to interested readers in this USERDA report. All of the calculations are concerned with whether a particle can grow to sufficient size, during impact extrusion, to act as dislocation multiplication sites.

\section{Computations Included in This Report Are:}

I. Carbide particle sizes attainable by diffusion controlled growth during impact extrusion.

II. Excess vacancy enhanced diffusivity during impact extrusion.

III. Enhanced diffusivity via dislocation "pipe" diffusion during impact extrusion. 
I. Carbide Particle Sizes Attainable by Diffusion Controlled Growth During Impact Extrusion.

Cahn ' has shown that heterogeneous nucleation of precipitates on dislocations is many orders of magnitude faster than homogeneous nucleation. Consequently, in the presence of a highly dislocated structure such as is formed during impact extrusion, nucleation would not limit precipitate formation. Instead, diffusion controlled growth would be the limiting process.

In order to arrive at a reasonably accurate upper bound on the size a precipitate particle could reach within the duration of deformation in impact extrusion, Zener's $s^{2}$ theory was employed. It is well known that his theory gives an overestimate of particle size in the small particle size range where capillarity should be considered. Thus, Zener's theory should provide a liberal upper bound in the particle size range of concern.

The details of Zener's treatment are given in his paper; only a brief outline is presented here. The treatment begins in a general way without invoking the dimensionality of growth. The particle size at any time, $t$, is given by:

$$
S=\alpha_{\lambda}(D t)^{\frac{1}{2}} \quad \text { Eq. } 1
$$

where $S$ is the growth coordinate or half-width for plate growth, the radius for cylindrical growth and the radius for spheres; $D$ is the diffusivity of the solute atoms and $\alpha_{\lambda}$ is a parameter called the growth coefficient which depends on the pertinent solute concentrations. $\alpha_{\lambda}$ is determined from the solution of the generalized partial differential equation (Zener's Eq. 5). The crux of the problem from this point on is to find the value of $\alpha_{\lambda}$ which satisties the following equation (Eq. 2): 


$$
\phi_{\lambda}\left(\alpha_{\lambda}\right)=\frac{2 \S}{\left(\alpha_{\lambda}\right)^{\lambda} \exp \left(\frac{\alpha_{\lambda}^{2}}{4}\right)}
$$

Eq. 2

where $\quad \xi=\frac{c_{\infty}-c_{\alpha}}{c_{\beta}-c_{\alpha}}$

$C_{\infty}=$ concentration of solute at a distance far from the precipitate (Fig. 1).

$C_{\beta}=$ concentration of solute in the precipitate (Fig. 1).

$C_{\alpha}=$ concentration of solute in the matrix in equilibrium with the precipitate (Fig. 1).

$$
\phi_{\lambda}(x)=x^{\rho^{\infty}} z^{1-\lambda} \exp \left(\frac{-z^{2}}{4}\right) d z
$$

Eq. 3

and $\lambda$ has the values 1,2 or 3 for plate growth, radial growth of a cylinder or spherical growth respectively.

a.) For $\lambda=1$ (plate), Eq. 2 becomes:

$$
\sqrt{\pi}\left[1-\operatorname{erf}\left(\frac{\alpha}{2}\right)\right]=\frac{2 \S}{\alpha_{1} \exp \left(\alpha_{1}{ }^{2} / 4\right)}
$$

where erf is the error function tabulated in Jahnke and Emde ${ }^{3}$ or other mathematical tables.

b.) For $\lambda=2$ (cylinder), Eq. 2 becomes:

$$
-\frac{1}{2} E i\left(-\frac{\alpha_{2}^{2}}{4}\right)=\frac{2 \S}{\alpha_{2}^{2} \exp \left(\frac{\alpha_{2}^{2}}{4}\right)}
$$


where $E i$ is the logarithmic integral function tabulated in Jahnke and Emde ${ }^{3}$. c.) For $\lambda=3$ (sphere), Eq. 2 becomes:

$$
\frac{1}{4} \Gamma\left(-\frac{1}{2}, \frac{\alpha_{3}^{2}}{4}\right)=\frac{2 \xi}{\alpha_{3}^{3} \exp \left(\frac{\alpha_{3}^{2}}{4}\right)}
$$

Eq. 2c

where $\Gamma(m, n)$ is the incomplete gamma function tabulated in Gradshteyn and Ryzhik ${ }^{4}$. Eq. $2 c$ could also be written as:

$$
\frac{4}{\alpha_{3}} \exp \left(\frac{-\alpha_{3}^{2}}{4}\right)-2 \sqrt{\pi}\left[1-\operatorname{erf}\left(\frac{\alpha_{3}}{2}\right)\right]=\frac{2 \xi}{\alpha_{3}^{3} \exp \left(\frac{\alpha_{3}^{2}}{4}\right)}
$$

Zener $^{2}$ has provided graphical representations of $\alpha_{1}$ and $\alpha_{3}$ as functions of composition parameters. However, he didn't provide this information for cylindrical growth, i.e., for $\lambda=2$. To be consistent with the assumption that nucleation could be neglected because it occurred on dislocations, the type of growth should be taken as cylindrical. Accordingly it was necessary to determine $\alpha_{2}$ for use in Eq. 2. The transcendental equation (Eq. 2b) was solved for various values of $\xi$ to obtain corresponding values of $\alpha_{2}$ and a graph of $\alpha_{2}$ versus $\xi$ was plotted as shown in Fig. 2 .

A value $\S=.06\left(C_{B} \approx .79, C_{\omega} \approx .1, C_{\alpha} \approx .06\right)$ is appropriate for the case of $440 \mathrm{~A}$ stainless steel which was equilibrated at about $1900^{\circ} \mathrm{F}(1310 \mathrm{~K}$ ) and extruded at some lower temperature where $\mathrm{Cr}_{23} \mathrm{C}_{6}$ carbides precipitate in the deformed austenite. As may be seen in Fig. 2, the corresponding value of $\alpha_{2}$ is about 0.25 . Therefore, Eq. 1 for cylindrical growth reads: 


$$
S=0.25(D t)^{\frac{1}{2}}
$$

Eq. $1 \mathrm{~b}$

The diffusivity of chromium in austenite is given by:

$$
D=3.2 \times 10^{-3} \exp \left[\frac{-53,000}{R T}\right] \mathrm{cm}^{2} / \mathrm{sec}
$$

Eq. 4

For a temperature of $1590^{\circ} \mathrm{F}(1139 \mathrm{~K})$ the diffusivity is $D=2.5 \times 10^{-13} \mathrm{~cm}^{2} / \mathrm{sec}$.

This temperature was chosen because it corresponds to the experimental condition which provided peak strengthening (presumably by precipitation of $\mathrm{Cr}_{23} \mathrm{C}_{6}$ ). However, other conditions and the resulting particle radii are presented in Table I. The times used in obtaining the values of $(D t)^{\frac{1}{2}}$ tabulated in Table I are for the following conditions:

i) $t=4.8 \times 10^{-4}$ secs was based on the time required to produce a $2.54 \mathrm{~cm}$ extrudate.

ii) $t=2.5 \times 10^{-3}$ secs was the time required for complete extrusion of a billet; this is a highly liberal value.

It is clear from the results in Table I that particles of physically meaningful size are not generally formed during extrusion, especially at the peak strengthening temperature of $1590^{\circ} \mathrm{F}\left(1139^{\circ} \mathrm{K}\right)$.

Arbitrarily choosing a physically meaningful $S$ of $25 \AA^{\circ}$ for comparison, shows that diffusivities 8000 or 1600 times the real diffusivity are required for times of $4.8 \times 10^{-4} \operatorname{secs}$ and $2.5 \times 10^{-3}$ secs respectively. In other words, these are the diffusion enhancement factors required. The value of 8000 is probably more accurate than the 1600 value. The enhancement factor is the ratio of the effective diffusivity, $D_{\text {eff }}$ to the bulk diffusivity, $D$, under equilibrium conditions, $D$ eff $d$.

In summary, particles cannot grow to physically meaningful sizes during impact extrusion. This is even more apparent in view of the fact that Zener's ${ }^{2}$ 
theory, which was applied in these calculations, gives an increasingly exaggerated particle size as the size decreases.

\section{Excess Vacancy Enhanced Diffusion Impact Extrusion}

Balluffi and Ruoff ${ }^{5-7}$ demonstrated theoretically that plastic deformation at creep rates cannot produce vacancy enhanced diffusion. Basically the reason was that vacancies induced by plastic deformation at creep rates are annihilated so fast on the diffusion time scale that they do not substantially alter the average jump rate of the diffusing species.

However, large plastic deformations performed at the high rates typical of impact extrusion conditions conceivably could produce excess vacancy concentrations with lifetimes sufficient to enhance diffusion substantially. In order to evaluate this possibility the following calculations were performed. The calculations are similar in principle to those of Balluffi and Ruoff ${ }^{5-7}$, but somewhat simpler in that dislocation-climb vacancy production is ignored. However, the model provides exceedingly liberal estimations of the vacancy production rate às will become apparent.

Formally, the time rate of change of excess vacancies is given by

$$
\frac{d n}{d t}=\frac{d n^{+}}{d t}+\frac{d n^{-}}{d t}
$$

where $\frac{d n}{d t}=$ net excess vacancy production rate

$$
\begin{aligned}
& \frac{d n^{+}}{d t}=\text { excess vacancy production rate due to plastic deformation } \\
& \frac{d n^{-}}{d t}=\text { excess vacancy annihilation rate }
\end{aligned}
$$

The forms of $\frac{d n^{+}}{d t}$ and $\frac{d n^{-}}{d t}$ depend on the particular models chosen. Dragging of jogged screw dislocations was chosen fur the $\frac{\mathrm{dn}^{+}}{\mathrm{dt}}$ model and vacancy annihilation 
at dislocations was selected to model $\frac{d n^{-}}{d t}$. Compared to other vacancy forming models based on plastic deformation, the jog dragging concept provides the highest vacancy production rate.

IIa.) Vacancy Production Rate Derivation:

From Hornstra ${ }^{8}$ it is known that the number of point defects, $N$, (interstitiakies or vacancies) created per elementary jog on a single screw dislocation can be expressed in the following way:

$$
N=\frac{t}{\Omega}\left[\bar{b}_{1} \bar{b}_{2} \bar{V}\right]
$$

where $t=$ time of dislocation motion

$\Omega=$ atomic volume of the lattice atoms

$\bar{b}_{1}=$ Burgers vector of moving jogged screw dislocation

$\bar{b}_{2}=$ Burgers vector of the dislocation which when intersected by the moving dislocation created the jog.

$\bar{V}=$ velocity vector of the moving dislocation

A term that determines whether the point defects created by the jogged screw are vacancies or interstitialcies has been omitted because the model assumed produces only vacancies. Further assuming that $\bar{b}_{1}, \bar{b}_{2}$ and $\bar{V}$ form an orthogonal set of vectors allows Eq. 2 to be written in scalar form as:

$$
N=\frac{t}{\Omega} b^{2} v
$$

Eq. 7

The assumptions employed lead to an overestimate of the number of vacancies created because in real crystals the intersecting dislocations are probably never normal to one another and certainly never all in the pure screw orientation normal to the direction of motion. 
Now from the fundamental equation for strain rate in terms of dislocation concepts we have:

$$
\dot{\varepsilon}=\phi \rho b V \dot{ }
$$

Eq. 8

where $\dot{\varepsilon}=$ tensile strain rate

$\phi=$ constant $\approx 0.5$

$\rho=$ mobile dislocation density, dislocation length per unit volume of material

$b=$ Burgers vector magnitude

$V=$ dislocation velocity

Rearranging Eq. 8 and substituting into Eq. 7 gives:

$$
N=\frac{t b^{2}}{\Omega} \frac{\dot{\varepsilon}}{\phi \rho b}
$$

Let $j$ be the number of elementary jogs per centimeter of dislocation. This allows the atomic concentration of vacancies created per second to be expressed in the following way:

$$
\frac{\mathrm{dn}^{+}}{\mathrm{dt}}=\frac{\mathrm{bj} \dot{\varepsilon}}{\phi N_{0} \bar{\Omega}}
$$

where $N_{0}=$ number of lattice sites per cubic centimeter of material.

Russe 11 and Jaffrey ${ }^{9}$ and Hirsch and Warrington ${ }^{10}$ have shown that the number of jogs per centimeter of dislocation is equal to the square root of the dislocation density. Therefore,

$$
j=\rho^{\frac{1}{2}}
$$

Furthermore, Ham and Jaffrey ${ }^{11}$ and Wintenberger ${ }^{12}$ have observed an empirical relation between disloration density and strain, $c$. Taking an average value 
of their proportionality constant gives

$$
\rho=3 \times 10^{\prime \prime} \mathrm{cm} / \mathrm{cm}^{3} \varepsilon
$$

Combining Eq. 11 with Eq. 12 and substituting the result into Eq. 10 results in the following relation:

$$
\frac{d n^{+}}{d t}=\dot{\varepsilon}\left[\frac{3 \times 10^{11} \mathrm{~cm}^{-2} b^{2}}{\phi^{2} \Omega^{2} N_{0}^{2}}\right]^{\frac{1}{2}} \varepsilon^{\frac{1}{2}}
$$

Taking $\phi=0.5$ and $\Omega=N_{0}$ reduces this to:

$$
\frac{\mathrm{dn}^{+}}{\mathrm{dt}}=\dot{\varepsilon}\left(10^{6} \mathrm{~cm}^{-1} \mathrm{~b}\right) \varepsilon^{\frac{1}{2}}
$$

IIb.) Vacancy Annihilation Rate Derivation:

For vacancy annihilation at dislocations first order kinetics are assumed with the result that:

$$
\frac{d n^{-}}{d t}=-k n
$$

Eq. 14

Following Girifalco and Grimes ${ }^{13}$

$$
K=\frac{2 \pi D_{v} \dot{r_{j}}}{\ln \frac{r_{j}}{r_{0}}}
$$

where $\quad D_{v}=$ vacancy diffusivity

$$
\rho=\text { dislocation density }
$$

$2 r_{i}=$ dislocation spacing which is $u^{-\frac{1}{2}}$.

$r_{0}=$ radius of a vacancy sink which is taken as twice the Burgers vector, $2 b$. 
Making the indicated substitutions and using Eq. 12 gives:

$$
\frac{\mathrm{dn}^{-}}{\mathrm{dt}}=\left[\frac{1.2 \pi \mathrm{v}_{\mathrm{v}} \times 10^{11} \mathrm{~cm}^{-2} \varepsilon}{\ln \left(4.8 \mathrm{~b}^{2} \times 10^{12} \mathrm{~cm}^{-2} \varepsilon\right)}\right] \mathrm{n}
$$

Eq. 16

IIc.) Excess Vacancy Concentration:

Adding Eqs. 13 and 15 as required by Eq. 5 yields:

$$
\frac{d n}{d t}=\frac{\dot{\varepsilon}}{2.2}(A \varepsilon)^{\frac{1}{2}}+\frac{\pi D_{v}(A \varepsilon)}{4 b^{2} \ln (A \varepsilon)} n
$$

Eq. 17

where $\quad A=4.8 b^{2} \times 10^{12} \mathrm{~cm}^{-2}$

IId.) Application to Extrusion:

Since the strain and strain-rate vary with position, and therefore with time, in the extrusion in a complex way, integration of Eq. 17 would be untenable. Consequently the mean effective strains and strain-rates given by Avitzur ${ }^{14}$ for round-to-round extrusion are employed. His expressions for mean effective strain-rate, $\overline{\dot{\phi}}$, and mean effective strain, $\bar{\phi}$, respectively are:

$$
\begin{aligned}
& \overline{\dot{\phi}}=\frac{6 V_{f} \cdot\left(\frac{D_{o}}{D_{f}}\right)^{2} D_{f}^{2} f(\alpha)[(1+\cos \alpha)(\sin \alpha)]}{D_{o}^{3}-D_{f}^{3}} \ln \frac{D_{o}}{D_{f}} \\
& \bar{\phi}=2 f(\alpha) \ln \frac{D_{0}}{D_{f}}
\end{aligned}
$$

where $\alpha=$ semicone angle of the dic

$$
\begin{aligned}
D_{0} & =\text { billet diameter } \\
D_{f} & =\text { minimum die diameter or extrusion diameter } \\
f(\alpha) & =\text { tabulated values for different die geometries (pg. } 160 \text { Ref. 14) } \\
v_{f} & =\text { final ram velocity }
\end{aligned}
$$


For the extrusion conditions of concern in this report

$$
\begin{aligned}
\alpha & =45^{\circ} \\
f(\alpha) & =1.0159
\end{aligned}
$$

and Eqs. 18 and 19 become:

$$
\overline{\dot{\phi}}=\frac{7.36 \mathrm{D}_{0}^{2} V_{f} \ln \frac{D_{o}}{D_{f}}}{D_{o}^{3}-D_{f}^{3}}=\frac{3.63 D_{o}^{2} V_{f} \bar{\phi}}{D_{o}^{3}-D_{f}^{3}}
$$

Eq. $18 \mathrm{a}$

and $\bar{\phi}=2.03 \ln \frac{D_{Q}}{D_{f}}$

Eq. $19 a$

Now substituting $\overline{\dot{\phi}}$ for $\dot{\varepsilon}$. and $\bar{\phi}$ for $\varepsilon$ in $E q .17$ gives:

$$
\frac{d n}{d t}=\frac{1.65 D_{0}^{2} V_{f}(A \bar{\phi})^{3 / 2}}{A\left(D_{0}^{3}-D_{f}^{3}\right)}+\frac{0.79 D_{v}(A \bar{\phi})}{b^{2} \ln (A \bar{\phi})} n
$$

Letting $b=2.5 \times 10^{-8}$ clil which is within a factor of two for al1 metals, $\bar{A} \bar{\phi}$ becomes:

$$
A \bar{\phi}=6.09 \times 10^{-3} \ln \frac{D_{0}}{D_{f}}
$$

Therefore,

$$
\frac{d n}{d t}=\frac{0.26 D_{o}^{2} V_{f}\left(\ln \frac{D_{o}}{D_{f}}\right)^{3 / 2}}{D_{o}^{3}-1 D_{f}^{3}}+\frac{7.70 \times 10^{12} \mathrm{~cm}^{-2} D_{v} \ln \frac{D_{o}}{D_{f}}}{-5.10+\ln \left(\ln \frac{D_{o}}{D_{f}}\right)} n
$$

Eq. 21

and since $D_{o}, D_{f}, V_{f}$ and $D_{v}$ are independent of time for a given extrusion condition, we may set:

$$
K_{1}=\left[\frac{0.26 R^{3}(\ln R)^{3 / 2}}{\left(R^{3}-1\right)}\right] \cdot \frac{V_{f}}{D_{0}}
$$

and $\quad K_{2}=\left[\frac{7.70 \times 10^{12}(\ln R)}{\ln (\ln R)-5.10}\right] \cdot D_{v} \mathrm{~cm}^{-2}$ 
where $\quad R=\frac{D_{0}}{D_{f}}$

For simplicity in writing let

$$
f(R)=\left[\frac{0.26 R^{3}(\ln R)^{3 / 2}}{\left(R^{3}-1\right)}\right]
$$

and $\quad g(R)=\left[\frac{7.70 \times 10^{12}(\ln R)}{\ln (\ln R)-5.10}\right]$

Therefore,

$$
k_{1}=f(R) \cdot \frac{V_{f}}{D_{0}}
$$

and

$$
K_{2}=g(R) \cdot D_{v} \mathrm{~cm}^{-2}
$$

and

$$
\frac{K_{1}}{K_{2}}=h(R)-\frac{V_{f}}{D_{0} D_{v} \mathrm{~cm}^{-2}}
$$

where $\quad h(R) \equiv \frac{f(R)}{g(R)}$

Thus Eq. 2 becomes

$$
\frac{d n}{d t}=k_{1}+k_{2} n
$$

Eq. 22

which integrates to

$$
\begin{array}{rl}
n & =-\frac{k_{1}}{k_{2}}\left[1-\exp \left(k_{2} t\right)\right] \\
\text { or } \quad n & n-\frac{v_{f} c m^{2}}{D_{0} D_{v}} h(R)\left[1-\exp \left(D_{v} g(R) t \mathrm{~cm}^{-2}\right)\right]
\end{array}
$$

Graphs of $f(K), g(K)$, and $h(R)$ versus $R$ are shown as Figs. 3,4 and 5 respectively. For realistic values of the reduction of area by extrusion, say from 5 percent $(R=1.03)$ to 98 percent $(R=7.07)$, the function $f(R)$ is always positive and the functions $y(R)$ and $h(R)$ are always negative. 
In order to evaluate the excess vacancy concentration, the appropriate values of $V_{f}, D_{o}, R$, and $t$ from the experimental conditions must be inserted in Eq. 23a. Furthermore, the values of $g(R)$ and $h(R)$ corresponding to the particular extrusion conditions were picked off figs. 4 and 5. Finally, the values of $D_{v}$ corresponding to the experimental temperatures were calculated from:

$$
D_{v}=0.58 \exp \left(\frac{-32,000}{R T}\right)
$$

which was given by Bullough and Perrin ${ }^{15}$ for vacancy diffusion in austenitic stainless steel.

The diffusion enhancement ascribable to excess vacancies is given by:

$$
\frac{D_{\text {eff. }}}{D}=1+\frac{n}{n_{\text {eq }}}
$$

where $D_{\text {eff. }}$ effective diffusivity of a solute or lattice atom

$$
\begin{aligned}
D & =\text { bulk lattice diffusivity of the solute atom } \\
n & =\text { deformation induced excess vacancy concentration } \\
n_{\text {eq }} & =\text { thermal equilibrium vacancy concentration }
\end{aligned}
$$

The values of $n_{\text {eq }}$ appropriate to the experimental temperatures were calculated by assuming the vacancy formation energy was equal to its migration energy. Thus the thermal equilibrium concentration was determined from

$$
n_{\text {eq }}=\exp \left(\frac{-32,000}{R T}\right)
$$


The measured or otherwise determined values described above are given for the experimental extrusion conditions in Table II, along with the enhancement given by the ratio $n / n_{\text {eq }}$. For the experimental conditions employed the exponential term in Eq. 23a was insignificant. Deformation times of the order $10^{-6} \mathrm{sec}$. or vacancy diffusivities two orders of magnitude slower or a combination of these would have been required to observe an appreciable effect of the exponential term. However, the exponential term would only serve to reduce the deformation induced vacancy excess and the associated diffusion enhancement. Consequently, the choice of deformation time is of no consequence to the vacancy enhancement.

At this point, it is pertinent to review some of the assumptions and their affect on the computed enhancement factors, $n / n_{\text {eq }}$.

1. The assumptions reducing Eq. 6 to Eq. 7 required all dislocations to be of pure screw orientation at right angles to one another and that the direction of motion i.e., direction of $\bar{v}$, be such that each atomic distance moved would produce a vacancy for each jog. Bow-out between jogs on the moving screw dislocations would produce a force on the jog that would not be at right angles to the dislocation. Furthermore, the direction of motion would generally be at some angle less than $90^{\circ}$ to the moving dislocation. Also the intersecting screws would, in reality, not be at $90^{\circ}$ to one another. Finally, only a fraction of the dislocations would be in the screw orientation. These combined factors could easily produce an overestimate of $n$ by a factor of 8 to 10 .

2. Eq. 11 assumes that all the dislocations are of an orientation that will create vacancy-producing jogs. Realistically, one-fourth of 
these or less would be in the appropriate orientation. Therefore, an overestimate of about 2 in $j$ would be involved:

These two assumptions would lead to an overestimate of $k_{1}$ by a factor of about 16 to 20 .

3. Eq. 15 involves the dislocation density capable of acting as dislocation sinks. In fact, only about one-half of them are suitable.

Since Eq. 23 involves the ratio of $k_{1}$ to $k_{2}$, it is evident that the assumptions give a factor of 8 to 10 times more excess vacancies than realistically expected. Allowing for a factor of two for the neglected edge dislocation contribution by stress-forced climb still leaves a factor of 4 to 5 overestimate. Dividing the enhancement factors in Table II by four reduces all the values to a level at least a factor of four below the enhancement required in the determination of the size of carbide particles attainable during extrusion (see the previous section).

Furthermore, the extrusion condition which produced the highest enhancement factor did not cause strengthening and the conditions which lead to maximum strengthening produced very low enhancement factors.

In conclusion, it may be stated that large deformations performed at high strain rates appear to produce significant excess vacancies and corresponding enhancement factors but they are not sufficient to permit growth of particles to significant sizes during the extrusion cycle. 
III. Enhanced Diffusivity Via Dislocation "Pipe" Diffusion During Impact Extrusion Kinetics of particle growth depend on solute diffusivity, $D$, according to $S=\alpha_{\lambda}(D t)^{\frac{1}{2}}$

Eq. 1

as shown in the first section. The solute diffusivity in dislocation cores (pipe-diffusion) is thought to be much higher than in the bulk. Consequently, if solute atoms spend a significant portion of their time in dislocation cores, the effective diffusivity can be increased considerably. Therefore, the purpose of this section is to determine whether solute chromium atoms in $440 \mathrm{~A}$ stainless steel have a large enough binding energy to dislocations to guarantee they will segregate to dislocation cores and to determine whether they will reside in the dislocation cores long enough to undergo enough "pipe diffusion" to enhance the effective diffusivity.

According to Bilby ${ }^{16}$ the binding energy of a solute to an edge dislocation is given by:

$$
\Delta H_{B}=4 \mu b r_{0}^{3}\left|\frac{r_{0}-r_{1}}{r_{0}}\right|\left(\frac{\sin \theta}{r}\right)
$$

where $\mu=$ shear modulus of the bulk

$b=$ Burgers vector magnitude

$r_{0}=$ radius of the solvent atoms

$r_{1}=$ radius of the solute atoms

$r=$ radial distance from the center of the dislocation core to a solute atom.

$\theta=$ angular portion of the solute atom with respect to the dislocation core 
From McEvily, Bush, Schaller and Schmatz ${ }^{17}$ :

$$
\begin{aligned}
& r_{0}=1.26 \AA \text { (Fe) } \\
& \left.r_{1}=1.28 \AA \text { (Cr }\right)
\end{aligned}
$$

Taking $r=2 b$ and the position of maximum binding as $\theta=3 \pi / 2$ gives

$$
\Delta H_{B}=6.3 \times 10^{-26} \mu
$$

The appropriate value of $\mu$ for F.C.C. iron at $1139 \mathrm{~K}$ (the temperature of peak strengthening) must be estimated. Using a value of $C_{44}$ for F.C.C. in alloys at room temperature ${ }^{18}$ and the temperature coefficient typical of F.C.C. copper and aluminum, the estimated value of $\mu$ was $8 \times 10^{11}$ dynes $/ \mathrm{cm}^{2}$. Therefore;

$$
\Delta H_{B}=5.1 \times 10^{-14} \mathrm{ergs}
$$

The concentration of solute in the dislocation core, $c_{d}$, where $r=2 b$, is given by

$$
C_{d}=C_{0} \exp \left[\frac{\Delta H_{B}}{k T}\right]
$$

Eq. 28

where $C_{0}$ is the concentration of solute in the bulk. At the temperature of peak strengthening, $1139 \mathrm{~K}$, for the binding energy computed above, the concentration of solute in the dislocation core is:

$$
c_{d}=1.4 c_{0}
$$

This value of dislocation core concentration, although not large, indicates there is a weak tendancy for solute chromium atoms to segregate to dislocations.

Since solute chromium has been shown to have a tendency to segregate to dislocation cores, it must be ascertained whether they can remain in the dislocation cores long enough to make a significant number of jumps in the core to enhance the diffusivity. The answer to this question can be approached by evaluating the maximum strain rate which will permit a solute atmosphere to keep pace with it. 
According to Ham and Jaffrey ${ }^{11}$ the critical strain rate is given by:

$$
\dot{\varepsilon}_{C}=\frac{4 b \rho D}{\ell}
$$

where $\dot{\varepsilon}_{c}$ the critical strain rate which is replaced by the average

effective strain rate in extrusion, $\overline{\dot{\phi}}_{C}$ (Eq. 18).

$\mathrm{b}=$ the Burgers vector $=2.5 \times 10^{-8} \mathrm{~cm}$

$\rho=$ the dislocation density to be replaced by $\rho=3 \times 10^{11} \bar{\phi}$ ( $\phi$ given by Eq. 19)

$D=$ the solute diffusivity $=2.5 \times 10^{-13} \mathrm{~cm}^{2} / \mathrm{sec}$ for chromium at $1139 \mathrm{~K}$ (Eq. 4)

$\ell=$ the extent of the atmosphere from the center of the dislocation core, which can be taken, for a liberal estimate, as $\ell=2 b$.

Therefore, Eq. 29 becomes

$$
\bar{\phi}_{C}=0.15 \mathrm{sec}^{-1} \bar{\phi}
$$

The average effective strain, $\bar{\phi}$, for the peak strengthening extrusion condition was

$$
\bar{\phi}=2.03\left|\ln \frac{1}{1.414}\right|=0.7
$$

Thus $\bar{\phi}_{\mathrm{C}}=0.1 \mathrm{sec}^{-1}$

This computed value of $\bar{\phi}_{C}$ should be compared with the experimental average effective strain rate of $745 \mathrm{sec}^{-1}$. Clearly, the experimental value of strain rate is almost $10^{4}$ times the critical value at which solute atoms could reamin with the dislocations.

It must be concluded, from these computations, that "pipe diffusion" of chromium would not contribute substantially to the rate of carbide precipitate growth under the conditions imposed by impact extrusion.

From the above considerations, it may be surmised that the dislocations 
in the present situation will not be solute enriched nor would solutes be able to move along with the dislocations. Consequently, dislocation pipe diffusion of the solute may be dealt with by considering only normal lattice concentrations of solute.

According to the treatment of $\mathrm{Hart}^{19}$ as presented by Shewmon ${ }^{20}$, the diffusivity enhancement, $\frac{D_{\text {eff }}}{D}$, for "pipe" diffusion is:

$$
\frac{D_{\text {eff }}}{D}=1+\frac{D_{p}}{D} g
$$

where: $D_{\text {eff }}=$ apparent diffusivity

$D=$ normal lattice diffusivity

$D_{p}=$ "pipe" diffusivity

$g$ = fraction of time an atom spends in the dislocation compared to time spent in the normal lattice.

A difficulty associated with this analysis is that values for $D_{p} / D$ are not available. However, Shewmon ${ }^{20}$ (p. 177 of Ref. 20) points out that for common crystal structures values at $D_{p} / D$ should be about the same at equal values of $T / T m$. He gives values of $D_{p} / D$ versus $T / T m$ for silver self-diffusion.

$\begin{array}{ll}\frac{\mathrm{D}_{\mathrm{P}} / \mathrm{D}}{8 \times 10^{5}} & \frac{T / \mathrm{Tm}}{2} \\ 9 \times 10^{6} & 0.7 \\ 2.7 \times 10^{8} & 0.6 \\ 4.5 \times 10^{10} & 0.4\end{array}$

For $0.4<\mathrm{T} / \mathrm{Tm}<0.5$ (appropriate to the present experimental conditions) it would appear that $D_{p} / D \simeq 10^{9}$ would be an appropriate choice and therefore: 


$$
\frac{D_{\text {eff }}}{D}=1+10^{9} \mathrm{~g}
$$

The earlier calculations indicated the enhancement, $\frac{D_{\text {eff }}}{D}$, required to obtain a particle with a $25 \AA$ radius under the impact extrusion conditions would be about 8000 or more. Accordingly g must be of the order $10^{-5}$. According to Shewmon 20

$$
g=\rho\left(\frac{1}{10^{15} \text { atoms } / \mathrm{cm}^{2}}\right)\left(\frac{10 \text { atoms }}{\text { disloc }}\right)=\rho\left(10^{-14} \mathrm{~cm}^{2} / \text { dis } 10 \mathrm{c} .\right)
$$

Thus, values of $\mathrm{g}$ near $10^{-5}$ or higher are expected for $\rho>10^{9} / \mathrm{cm}^{2}$ which is certainly the case under impact extrusion conditions.

However, more recently Wazzan and Dorn ${ }^{21}$ examined pipe diffusion enhancement and concluded that static dislocations did not enhance diffusivity. They showed, on the other hand, that enhancement was produced by moving dislocations and it increased with strain rate.

Demonstrating that this enhancement could not be attributed to excess vacancies they turned to Hart's ${ }^{19}$ analysis of pipe diffusion in a modified form:

$$
\frac{D_{\text {eff }}}{D}=1+g^{\prime} D_{p} / D
$$

They could not give a definite expression for ' $g^{\prime}$, but suggested it would be proportional to $\dot{\varepsilon}$ (through dislocation velocity) since they visualized the diffusing atoms would be bumped into by the moving dislocations and the concentration gradient in the pipe would be greater as the dislocation velocity increased. This might be true for their experiment (radioactive $\mathrm{Ni}$ in $\mathrm{Ni}$ ) and at their low strain rates or if a substantial binding energy between a solute and dislocation existed. However, in rather dilute solutions and with high dislocation velocities it seems rather improbable that the concentration would change much. 
The analysis on the following pages shows that a value of $\mathrm{g}$ for moving dislocations is independent of dislocation velocity but is proportional to dislocation density. The model is shown schematically in Figs. 6 and 7 . It involves a regular distribution of dislocations moving uniformly in one direction with a velocity, $V$. This is equivalent to the dislocations being considered as stationary with the solutes moving in the opposite direction with the same speed as the dislocations. The physical picture is one in which the solutes, moving by random walk with a superimposed drift toward a growing particle, periodically enter a dislocation core and exercise more rapid jumps toward the particle before the dislocation passes by. The net effect is an increased rate of drift toward the particles. However, only those solute atoms in the dislocation path are affected in this model.

A solute atom in the path of a dislocation pipe, $P$, which is $R_{0}$ wide will spend time, $t_{1}$, in the lattice in the dislocation affected zone, $D A Z$, and time, $t_{d}$, in the pipe where:

$$
\begin{gathered}
t_{1}=\left(\frac{\rho^{-\frac{1}{2}}-R_{0}}{V}\right) \\
t_{d}=\frac{R_{0}}{V}
\end{gathered}
$$

Therefore,

$$
g=\frac{t_{d}}{t_{1}}=\frac{R_{0} \rho^{\frac{1}{2}}}{1-R_{0} \rho^{\frac{1}{2}}}
$$

However, this ratio only includes those solutes in the dislocation path; those outside the dislocation path (in the BDZ) will spend no time in the "pipe". Consequently, if a solute-concentration weighted average is taken, the following average time ratio is obtained: 


$$
\begin{gathered}
\frac{\overline{t_{d}}}{t_{1}}=\rho^{\frac{1}{2}} R_{0}\left(\frac{t}{t_{1}}\right)+\rho^{\frac{1}{2}}\left(\rho^{-\frac{1}{2}}-R_{0}\right) \cdot 0 \\
=\rho^{\frac{1}{2}} R_{0}\left(\frac{R_{0} \rho^{\frac{1}{2}}}{1-R_{0} \rho^{\frac{1}{2}}}\right) \\
=\frac{\rho R_{0} 2}{1-R_{0} \rho^{\frac{1}{2}}}
\end{gathered}
$$

Since the product $R_{0} \rho^{\frac{1}{2}}$ is of the order $10^{-4}$, it may be neglected with respect to unity. Thus,

$$
\frac{\overline{t_{d}}}{t_{1}}=\rho R_{0}^{2}
$$

With $R_{0} \sim 4 b$ this yields

$$
g=\left(\frac{\overline{t_{d}}}{t_{1}}\right)=10^{-14} \rho\left(\rho \text { in } \mathrm{cm} \cdot{ }^{-2}\right)
$$

which is identical to the static case which suggests enhancements, $\frac{D \text { eff }}{D}=1+g \frac{D}{D}$, of the order $10^{6}$. It must be admitted, therefore, that pipe diffusion might help particles reach $25 \AA$ radii during impact extrusion.

However, some experimental results negate the above conclusion. According to the data presented by Shewmon ${ }^{20}$ for $D_{p} / D$ ratios, the ratio increases extremely rapidly with decreasing temperature. Accordingly, lower temperature deformation (which will probably result in greater dislocation density for a particular strain) should provide substantially greater enhancement. Experimental data reveal, to the contrary, that the lowest temperature extrusion was not strengthened.

In conclusion, dislocation pipe diffusion under impact extrusion conditions could enhance particle growth. However, the apparent experimental growth characteristic did not follow the behavior pattern that pipe diffusion would predict. 
IV. OVERALL SUMMARY

In Section I of this report Zener's theory of particle growth was applied, after determining the growth coefficient for cylinders, to the case of $\mathrm{Cr}_{23} \mathrm{C}_{6}$ carbides during impact extrusion of $440 \mathrm{~A}$ stainless steel. It was shown that these carbide particles cannot grow to physically meaningful sizes in the duration of extrusion when bulk diffusion controls. In order for particles to grow to $25 \AA$ radius in the extrusion duration it was shown that diffusion enhancements of the order of 8000 and probably greater would be necessary.

Section II dealt with the possibility that deformation-induced excess vacancies would provide the diffusion enhancements determined in Section I. It was demonstrated that diffusion may be enhanced somewhat by deformationinduced excess vacancies during impact extrusion, but the degree of enhancement was negligible compared to the degree required.

Finally, Section III examined the possibility that dislocation "pipe" diffusion might provide the required enhancements. Within the framework of the relatively pourly developed analyses of "pipe" diffusion, it was shown that, in principle, "pipe" diffusion could provide the enhancements required. However, the experimental observations were in disagreement with the predictions of this model.

Consequently, it is concluded that $\mathrm{Cr}_{23} \mathrm{C}_{6}$ precipitates cannot grow to physically meaningful sizes in the duration of impact extrusion. 


\section{REFERENCES:}

1. J. W. Cahn: Acta Met. (1967) $5,169$.

2. C. Zener: J. Appl. Phys. (1949) 2ㅁ, 950.

3. E. Jahnke and F. Emde: Tables of Functions, 4th Edition, Dover Publications (1945).

4. I. S. Gradshteyn and I. M. Ryzhik: Table of Integrals, Series, and Products, 4th Edition, Academic Press (1965).

5. R. W. Balluffi and A. L. Ruoff: J. Appl. Phys. (1963) 34, 1634.

6. A. L. Ruoff and R. W. Balluffi: J. Appl. Phys. (1963) $34,1848$.

7. A. L. Ruoff and R. W. Balluffi: J. Appl. Phys. (1963) $34,2862$.

8. J. Hornstra: Acta Met. (1962) 10, 987.

9. B. Russell and D. Jaffrey: Acta Met. (1965) $1 \underline{1}, 1$.

10. P. B. Hirsch and D. H. Warrington: Phil. Mag. (1961) $7,735$.

11. R. K. Ham and D. Jaffrey: Phi1. Mag. (1967) 15, 247.

12. M. Wintenberger: C. R. Acad. Sci., Paris (1957) 2244, 2800.

13. L. A. Girifalco and H. H. Grimes NASA TR-R-38 (1959), Supt. Docs., U.S. Govm't. Printing Office, Washington D.C.

14. B. Avitzur: Metal Forming: Processes and Analysis, (1968), McGraw-Hi11, New York, 195 et seq.

15. R. Bullough and R. C. Perrin: Radiation-Induced Voids in Metals, U.S. A.E.C., (1972), pg. 769.

16. B. A. Bilby: Proc. Royal Soc., (1950) A63, 191.

17. A. J. McEvily, Jr., R. H. Bush, F. W. Schaller and D. J. Schmatz: Trans. ASM (1963) $\underline{56} ; 753$

18. F. X. Kayser: Private Communication, Iowa State University, 1975.

19. E. Harl: Acta Met. (1957) 5 , 597.

20. P. G. Shewmon: Diffusion in Solids, McGraw-Hill (1963).

21. A. R. Wazzan and J. E. Dorn: J. Appl. Phys. (1965) 36, 222. 
TABLE I : VALUES OF $(D t)^{\frac{1}{2}}$ FOR THE EXPERIMENTAL CONDITIONS EMPLOYED

\begin{tabular}{|c|c|c|c|c|}
\hline \multirow[b]{2}{*}{$T_{A}=\frac{1}{2}\left(T_{M}+T_{E}\right),{ }^{\circ} F\left({ }^{\circ} K\right)$} & \multirow[b]{2}{*}{$\mathrm{D}, \mathrm{cm}^{2} / \mathrm{sec}$} & \multicolumn{2}{|c|}{$\sqrt{D t}, \AA$} & \multirow{2}{*}{ *S, } \\
\hline & & $t=4.8 \times 10^{-4} \operatorname{secs}$ & $t=2.5 \times 10^{-3} \operatorname{secs}$ & \\
\hline $1371(1017)$ & $1.5 \times 10^{-14}$ & 0.3 & 0.6 & $<1$ \\
\hline $1590(1139)$ & $2.5 \times 10^{-13}$ & 1.1 & 2.5 & $<1$ \\
\hline $1820(1266)$ & $2.6 \times 10^{-12}$ & 3.5 & 8.1 & $<1,2$ \\
\hline $1486(1081)$ & $7.2 \times 10^{-14}$ & 0.6 & 1.3 & $<1$ \\
\hline $1725(1214)$ & $1.7 \times 10^{-12}$ & 2.3 & 5.2 & $<1,1,3$ \\
\hline $1890(1305)$ & $4.9 \times 10^{-12}$ & 4.8 & 11.1 & $1.2,2.8$ \\
\hline $1551(1117)$ & $1.6 \times 10^{-13}$ & 0.9 & 2.0 & $<1$ \\
\hline $1762(1234)$ & $1.5 \times 10^{-12}$ & 2.7 & 6.1 & $<1,1.5$ \\
\hline $2050(1394)$ & $1.8 \times 10^{-11}$ & 9.3 & 21.2 & $2.3,5.3$ \\
\hline
\end{tabular}

* For an $\alpha_{2}$ value of 0.25 
TABLE II. VALUES OF EXTRIJSION PARAMETERS AND ENIIANCEMENT FACTORS FOR 440A STAINLESS STEEL

\begin{tabular}{|c|c|c|c|c|c|c|c|c|c|c|}
\hline$T_{A}=\frac{1}{2}\left(T_{M}+T_{E}\right),{ }^{\circ} \mathrm{F}\left({ }^{\circ} \mathrm{K}\right)$ & $\mathrm{D}_{0}, \mathrm{~cm}$ & $R \equiv \frac{D_{0}^{0}}{D_{f}}$ & $D_{v} \times 10^{7}, \frac{\mathrm{cm}^{2}}{\mathrm{sec}}$ & $\frac{v_{f}}{D_{v}} \times 10^{-9}, \mathrm{~cm}$ & $1^{-1} h(R) \times 10^{14}$ & $g(R) \times 10^{-11}$ & $t, \sec$ & $\mathrm{n} \times 10^{4}$ & $n_{e q} \times 10^{6}$ & $\frac{n}{n_{e q}}$ \\
\hline $1371(1017)$ & 2.54 & 1.414 & 0.83 & 4.28 & -18.9 & -4.5 & $4.8 \times 10^{-4}$ & 3.19 & 0.2 & 1595 \\
\hline $1590(1139)$ & $"$ & $"$ & 4.7 & 1.01 & $"$ & $"$ & $"$ & 0.75 & 0.8 & 94 \\
\hline $1820(1266)$ & $"$ & $"$ & 19.5 & 0.16 & $"$ & $"$ & $"$ & 0.12 & 3.0 & 4 \\
\hline $1486(1081)$ & $"$ & 2 & 2.2 & 1.5 & -17.6 & -9.7 & $"$ & 1.04 & 0.4 & 260 \\
\hline $1725(1214)$ & $"$ & $"$ & 11.5 & 0.37 & $"$ & $"$ & $"$ & 0.26 & 1.9 & 14 \\
\hline $1890(1305)$ & $"$ & $"$ & 28.0 & 0.13 & $"$ & $"$ & $"$ & 0.09 & 4.5 & 2 \\
\hline $1551(1117)$ & $"$ & 4 & --- & --- & -19.2 & -22.2 & $"$ & --- & -- & -- \\
\hline $1762(1234)$ & $"$ & $"$ & 14.5 & 0.42 & $"$ & $"$ & $"$ & 0.32 & 2.4 & 13 \\
\hline $2050(1394)$ & $"$ & $"$ & 59.0 & 0.1 & $"$ & $"$ & $"$ & 0.07 & 11.0 & 1 \\
\hline
\end{tabular}




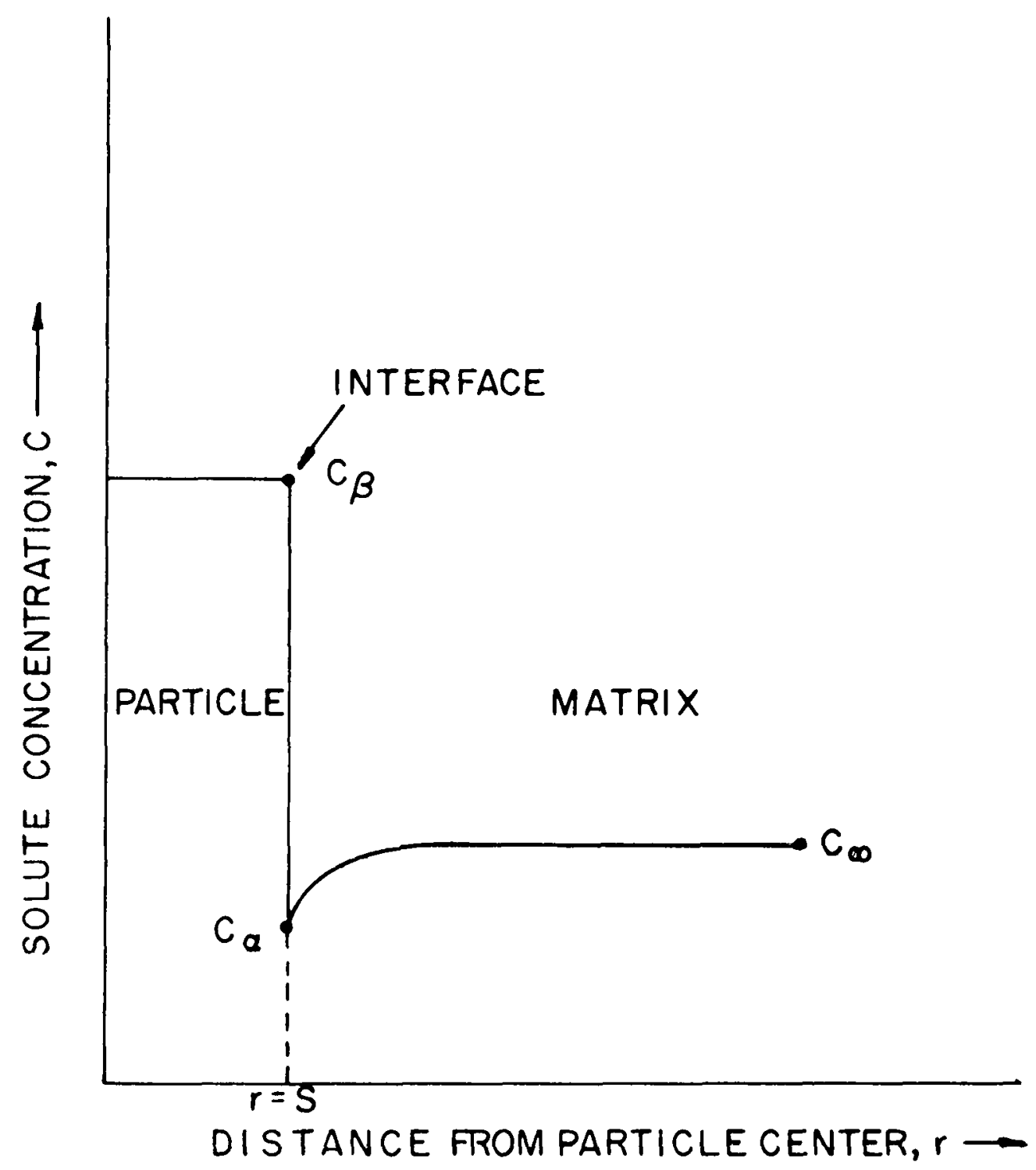

Fig. 1. A schematic illustration of the solute concentration distribution pertinent to alloy carbide growth. 


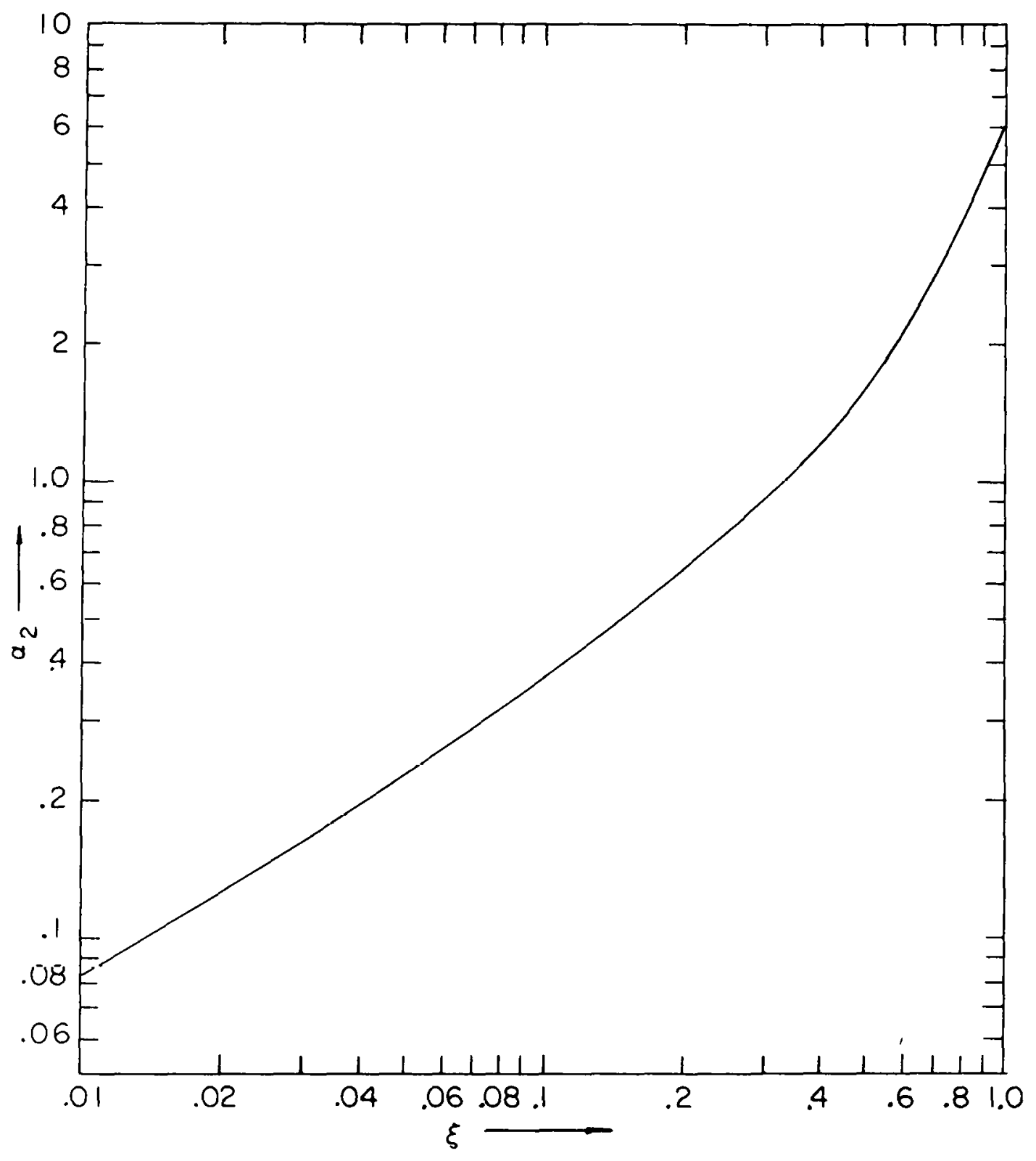

Fig. 2. A plot of the cylindrical growth cocfficient, $\alpha_{2}$, versus the composition parameter, $\xi$, based on Zener's ${ }^{2}$ theory. 


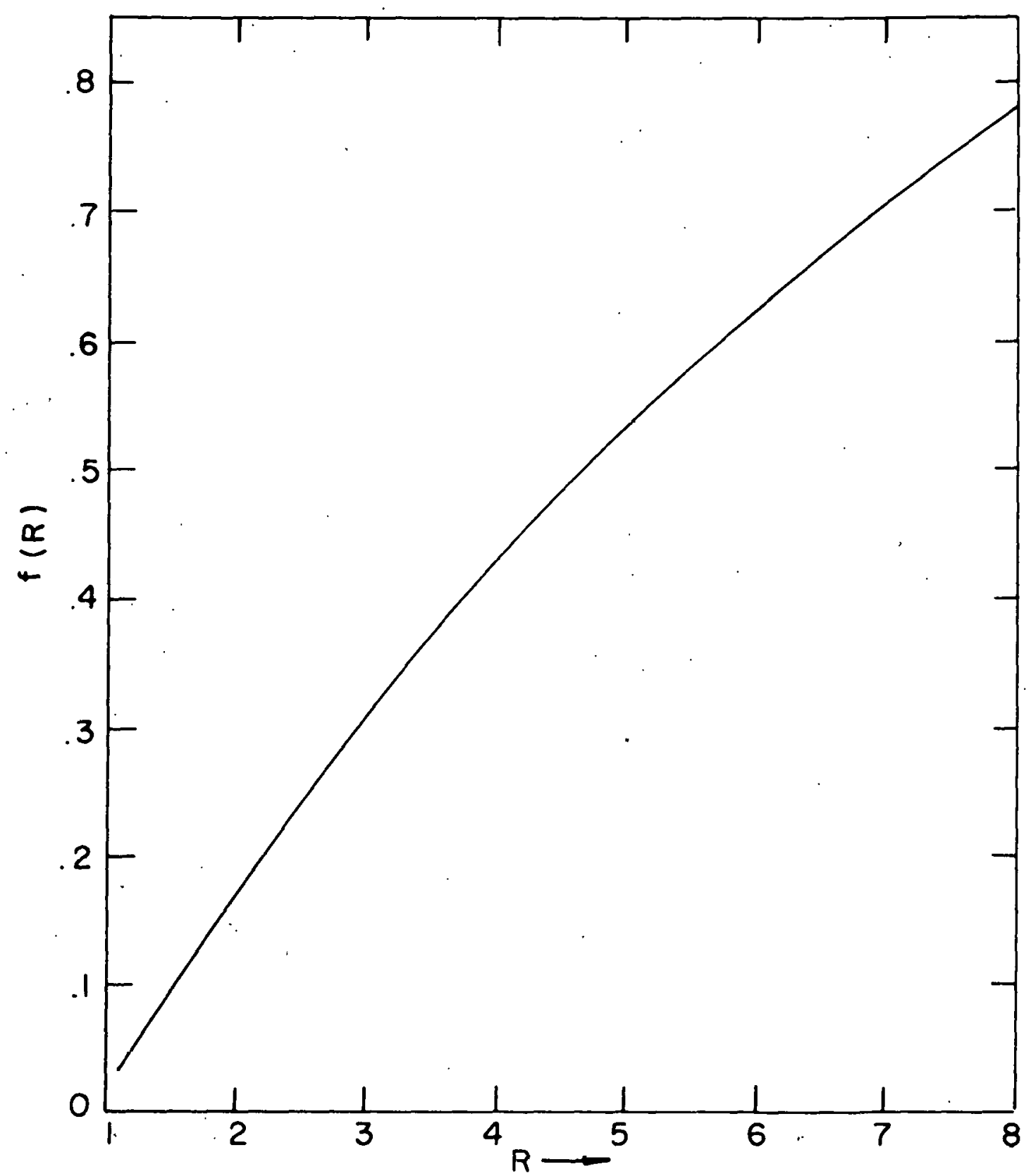

Fig. 3. A plot of the function $f(R)$ versus $R$. 


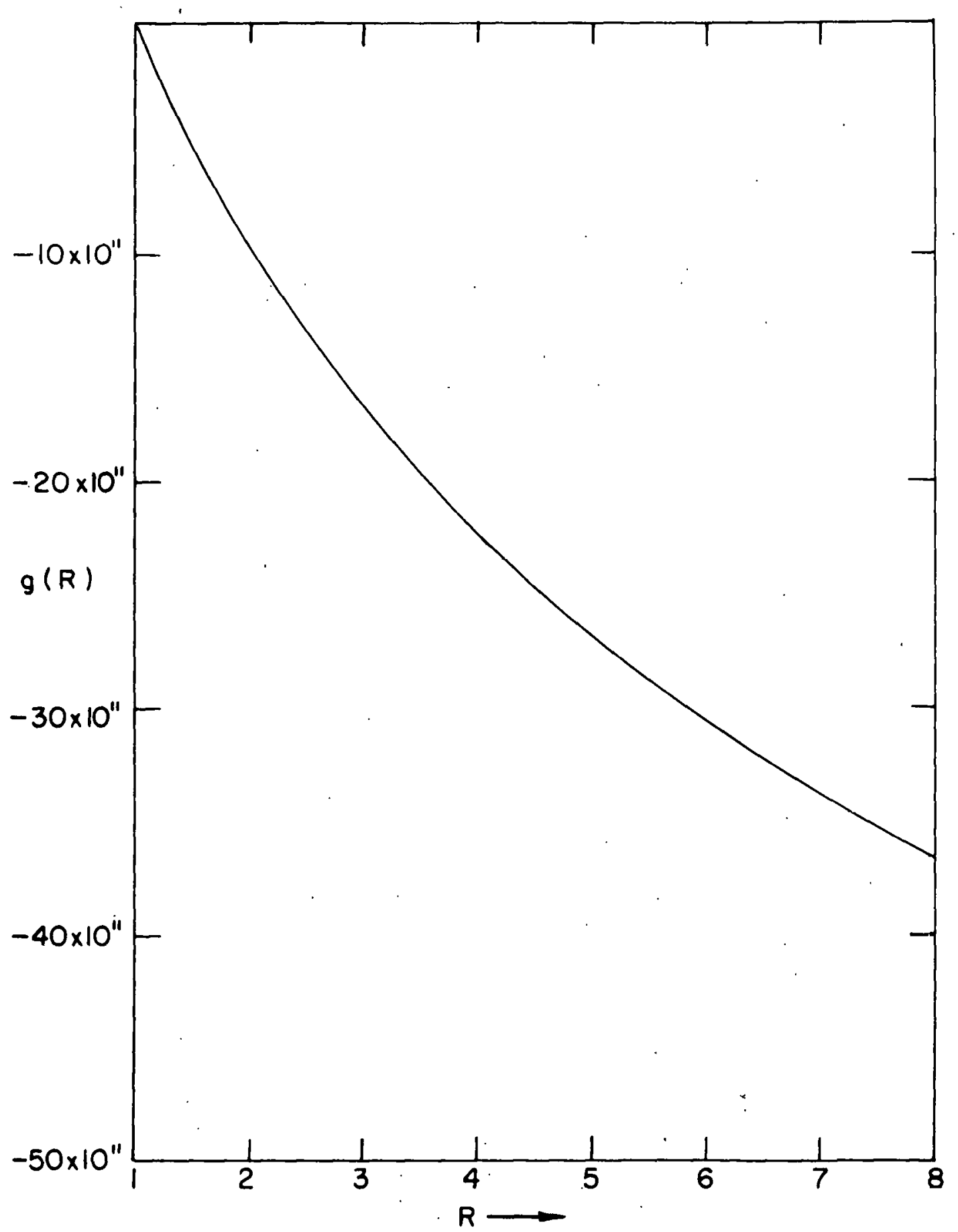

Fig. 4. A plot of the function $g(R)$ versus $R$. 


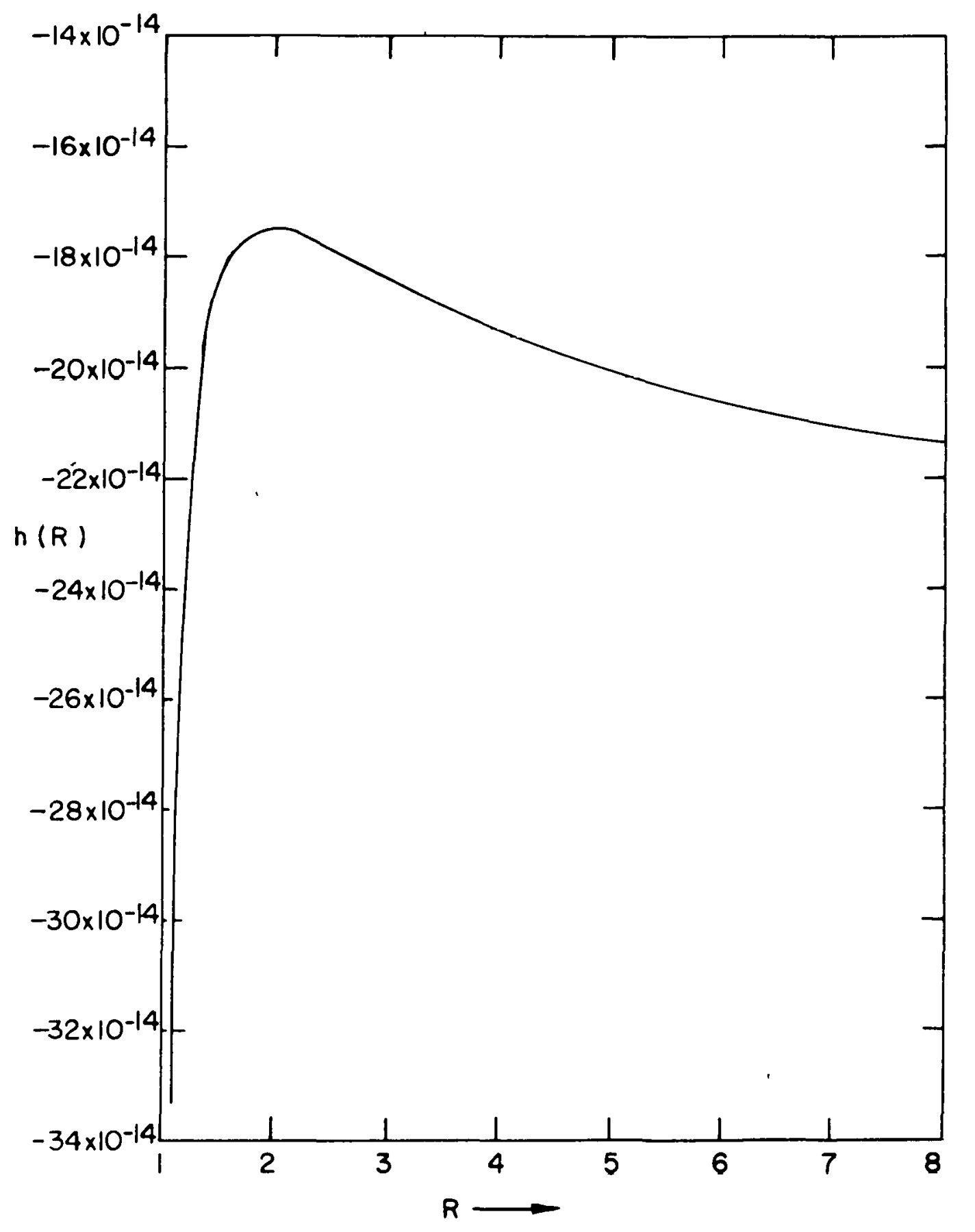

Fig. 5. A plot of the function $h(R)=f(R) / g(R)$ versus $R$. 


\section{END VIEW}

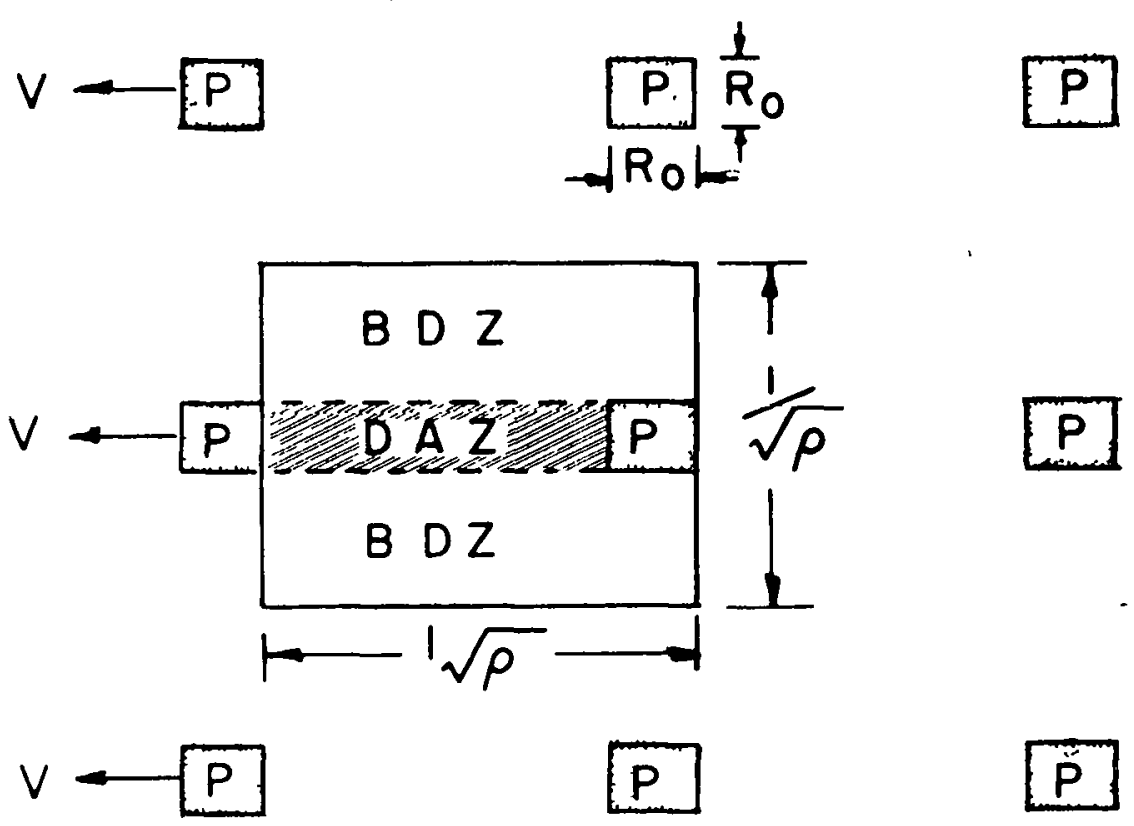

SYMBOLS

P DISLOCATION PIPE

$B D Z=$ BULK DIFFUSION ZONE

$D A Z=$ DISLOGATION AFFECTED ZONE

$P=$ DISLOCATION DENSITY, $\mathrm{cm}^{-2}$

AREAS PER DISLOCATION

$$
\begin{aligned}
& A_{P}=R_{0}^{2} \\
& A_{B D Z}=\rho^{-\frac{1}{2}}\left(\rho^{-\frac{1}{2}}-R_{0}\right) \\
& A_{D A Z}=R_{0}\left(\rho^{-\frac{1}{2}}-R_{0}\right) \\
& A_{\text {TOTAL }}=1 / \rho
\end{aligned}
$$

Fig. 6. A schematic 'End View' illustration of the model used in analyzing "pipe" diffusion of moving dislocations. 


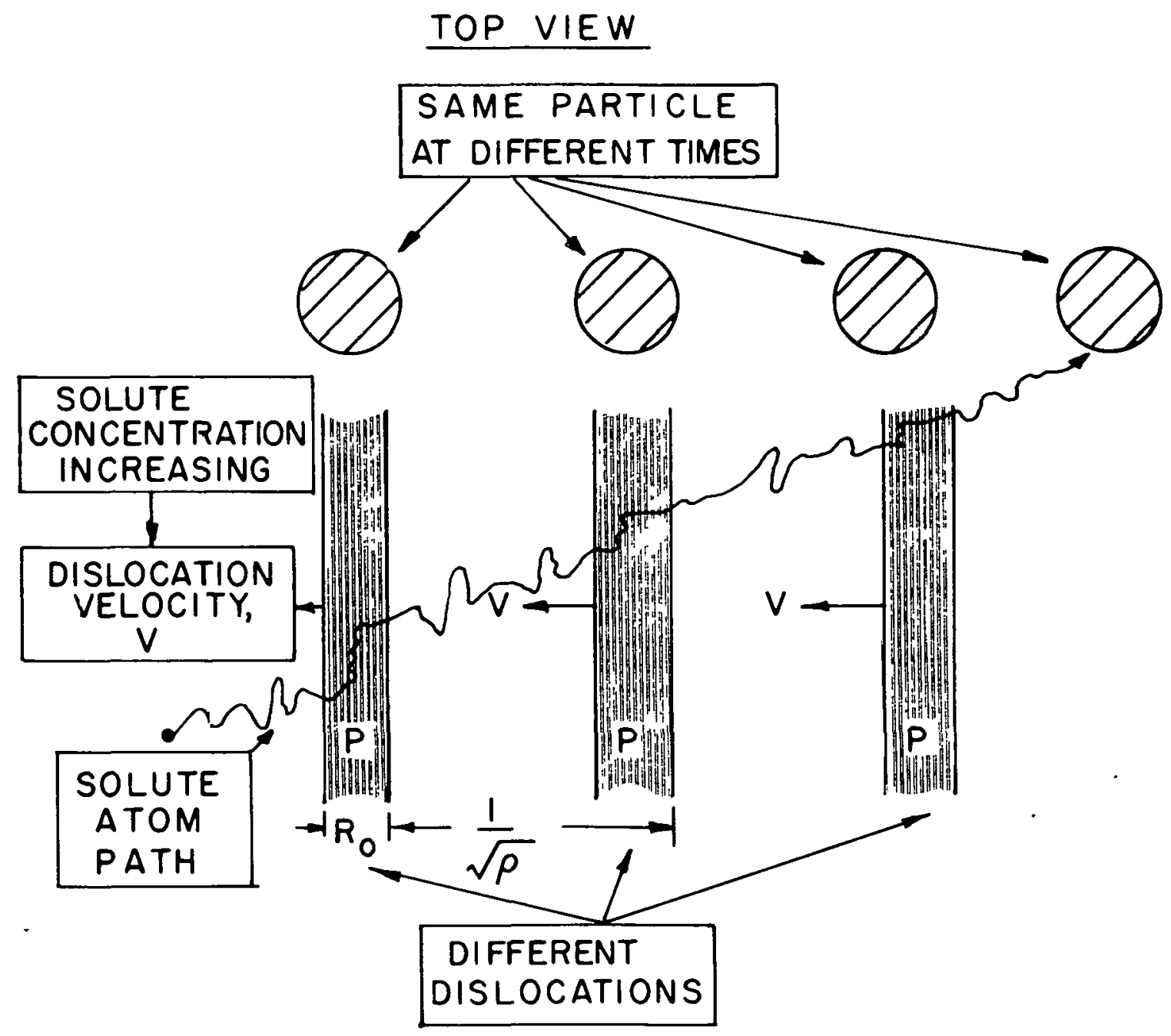

Fig. 7. A schematic 'Top View' illustration of the model used in analyzing "pipe" diffusion of moving dislocations. 